\title{
Experimental Evidence Relating Charge Transfer State Kinetics and Strongly Reduced Bimolecular Recombination in Organic Solar Cells
}

Nasim Zarrabi ${ }^{1}$, Oskar J. Sandberg ${ }^{*}$, Christina Kaiser ${ }^{1}$, Jegadesan Subbiah ${ }^{2}$, David J. Jones ${ }^{2}$, Paul Meredith $^{1^{*}}$ and Ardalan Armin ${ }^{1^{*}}$

[1] Sustainable Advanced Materials (Ser-SAM), Department of Physics, Swansea University, Singleton Park, Swansea SA2 8PP, United Kingdom

[2] Bio21 Institute and School of Chemistry, University of Melbourne, Parkville 3010, Australia

Email: ardalan.armin@swansea.ac.uk; o.j.sandberg@swansea.ac.uk; paul.meredith@swansea.ac.uk

\section{Abstract}

Charge carrier mobility in organic bulk heterojunction solar cells is generally limited to $10^{-3} \mathrm{~cm}^{2} \mathrm{~V}^{-1} \mathrm{~s}^{-1}$ or so. This constrains the charge-extraction rate and the practical range of junction thicknesses. High efficiencies have been achieved using junctions as thick as a micron in some limited material systems in which charge carrier mobilities are found to be "ordinary" but where the bimolecular recombination rate constant is significantly reduced relative to the diffusion limit. This strategy for reducing the recombination may be a way forward for industrial scaling of organic solar cells provided a structureproperty relation can be found to deliver reduced recombination. Current studies suggest that the main mechanism of reduced recombination should be due to the fast dissociation of the interfacial charge transfer states to free charges with respect to their decay rate to the ground state. However, whether reduced recombination is due to the suppressed decay rate or improved dissociation rate has remained a matter of some conjecture. In this work, we use a recently introduced donor-acceptor system which exhibits significantly reduced recombination. Based on measurements of the kinetics of free charges and CT states, we provide experimental evidence that an increase of the dissociation rate of CT states upon solvent annealing is responsible for the reduced recombination. Morphological studies point towards purer and more percolated domains in the solvent annealed system which may, therefore, have a stronger entropic driving force for CT dissociation. These are important observations towards a full understanding of reduced recombination for material design and synthesis. 


\section{Introduction}

Emerging novel material systems based upon non-fullerene electron acceptors have improved the power conversion efficiency (PCE) of organic solar cells (OSC) up to $18 \% .{ }^{1}$ However, these efficiencies are limited to laboratory small area devices $\left(<1 \mathrm{~cm}^{2}\right)$. The upscaling of OSCs to make industrially viable modules is a challenging task and there are multiple problems to be overcome. ${ }^{2,3}$ One of the problems is related to the junction thicknesses; the photoactive layer of technologically relevant OSCs is usually deposited on a transparent electrode via solution processing of a donor-acceptor blend. While spin coating or similar methods are often used at the lab scale, commercializing large area OSCs requires industry-relevant high throughput deposition methods such as role-to-role processing. Inevitably, the active layer must be sufficiently thick to avoid defects that can partially short-circuit large area devices. This is challenging since the performance parameters of organic solar cells, particularly the shortcircuit current density $\left(U_{\text {sc }}\right)$ and fill factor $(\mathrm{FF})$, are highly sensitive to the junction thickness. ${ }^{4-6}$ It is wellknown that the main reason for the poor performance of thick junction OSCs is low charge collection efficiency due to: (1) a slow charge extraction rate associated with low free carrier mobilities; and (2) a fast bimolecular recombination rate within the bulk of the active layer. Despite considerable efforts by the community, the mobility of free carriers (electrons and holes) cannot be improved much beyond $10^{-3} \mathrm{~cm}^{2} \mathrm{~V}^{-1} \mathrm{~s}^{-1}$ in diode architectures relevant to solar cells, because of the disordered nature of organic semiconductors. Yet, a handful of material systems have shown promisingly high efficiencies even when the junction thickness is increased to a micron which is considered to be "very thick" in organic solar cell nomenclature.

Recombination of free charges in a material with low mobility can be generally described by the Langevin recombination rate which assumes that the effective recombination rate is determined by the encounter rate of free carries when the Coulomb radius is larger than the hopping distance. ${ }^{7}$ Since this process is diffusion-limited, it is dependent on the mobility of the free charge carriers. With a few exceptions aside, ${ }^{8,9}$ it has been shown that the recombination rate in pristine organic semiconductors can be more or less explained by Langevin recombination rate with a rate constant given by 
$\beta_{\mathrm{L}}=q\left(\mu_{\mathrm{p}}+\mu_{\mathrm{n}}\right) / \varepsilon \varepsilon_{0}$, where $\mu_{\mathrm{n}}$ and $\mu_{\mathrm{p}}$ are the electron and hole mobility, $q$ is the elementary charge, $\varepsilon$ is the dielectric constant and $\varepsilon_{0}$ the vacuum permittivity. Conversely, the experimentally determined bimolecular recombination rate constants in donor-acceptor bulk heterojunctions, $\beta_{\mathrm{Bulk}}$, is generally smaller than the value expected from the Langevin relation. The reduction factor relative to Langevin recombination rate can be define as:

$$
\gamma=\frac{\beta_{\mathrm{Bulk}}}{\beta_{\mathrm{L}}}
$$

A reduction factor of 0.1 is very common in most $\mathrm{BHJ}$ systems ${ }^{10}$ while only very few exceptional systems exhibits strongly reduced recombination. One classic example of a material system with suppressed recombination is thermally annealed $\mathrm{P} 3 \mathrm{HT}: \mathrm{PC}_{60} \mathrm{BM} .{ }^{11}$ This material system delivers a reduced recombination rate of $\sim 100$ times less than the Langevin rate $(\gamma=1 / 100)$, which allows for the FF and the PCE to remain optimal in a range of thicknesses from 80 to $180 \mathrm{~nm} \cdot{ }^{12}$ In 2014 , Sun et al. ${ }^{13}$ introduced the donor benzodithiophene terthiophene rhodanine (BTR) which delivered a reduction factor of $\gamma=1 / 150$ upon solvent vapour annealing (SVA) when blended with $\mathrm{PC}_{71} \mathrm{BM} .{ }^{14}$ This material was arguably the first high efficiency material system exhibiting reduced recombination. It was shown that a $350 \mathrm{~nm}$-thick junction $\mathrm{BTR}: \mathrm{PC}_{71} \mathrm{BM}$ solar cell could deliver a high $\mathrm{FF}(\sim 0.75)$ and a PCE of $9.5 \%$ as a result of the reduced recombination, even though the electron and hole mobilities remained relatively low and even imbalanced. In 2016, a Naphtho[1,2-c:5,6-c'] Bis([1,2,5]Thiadiazole)based $\pi$-conjugated polymer (NT812) was introduced by Jin et al. ${ }^{15}$ Following thermal annealing, a $800 \mathrm{~nm}$-thick junction of NT812: $\mathrm{PC}_{71} \mathrm{BM}$ was found to have a reduction factor of $\gamma=1 / 800$, achieving a PCE of $>9 \%{ }^{16}$

It is important to note that the suppressed recombination in these material systems is only significant if one or more post treatment processes such as thermal or solvent vapour annealing follow the active layer deposition. It has been shown that these treatments influence the active layer morphology, however, a clear structure-property relation is still lacking. In the case of $\mathrm{P} 3 \mathrm{HT}: \mathrm{PC}{ }_{60} \mathrm{BM}$, for example, thermal annealing increases crystallinity and hence the domain sizes in the bulk. ${ }^{17,18}$ As a result, the 
suppressed recombination rate was initially attributed to the geometrical separation (confinement) of electrons and holes within their respective domains. ${ }^{19}$ On the other hand, since electrons and holes do not have the same mobilities and are confined in separated domains, it has also been suggested that different arrival times of the electron and hole to the donor-acceptor interface may be responsible for the suppressed recombination rate. ${ }^{20,21}$ However, Kinetic Monte Carlo simulations for realistic domain sizes has revealed that this geometrical effect only becomes relevant when the domain sizes are extremely large (> $30 \mathrm{~nm}$ ) which is not generally the case in bulk heterojunction OSCs. ${ }^{22}$ For typical phase separations of 5-10 nm, in turn, it was found that the geometrical confinement of electrons and holes in their respective domains only suppresses the bimolecular recombination by a factor of 10 at most. This "geometrical" suppression of recombination can be formulated as $\gamma_{\mathrm{Geo}}=\frac{\beta_{\mathrm{en}}}{\beta_{\mathrm{L}}}$ where $\beta_{\mathrm{en}}$ is the encounter rate constant. In such cases, the more imbalanced the electron and hole mobility, the more reduced the encounter rate becomes as the faster carrier must wait at the interface to meet the slower carrier.

The above discussion is built around the kinetics of electrons and holes and their encounter rate in a bulk heterojunction. In general, however, the generation and recombination of electrons and holes in BHJs occurs via charge transfer (CT) states, acting as intermediate charge recombination/generation centres. In Figure 1 a schematic of the charge recombination mechanism is shown with its corresponding rates. This correlation between the charge generation quantum yield and the reduction factor has been recently confirmed, experimentally, using a meta-analysis by Shoaee et $a l .{ }^{10}$ The authors have shown that the reduction factor depends on the kinetics of the CT states and more specifically on the ratio between dissociation rate constant of the CT states to free carriers $k_{\mathrm{d}}$ as well as the decay rate constant of the CT state to the ground state $k_{\mathrm{f}}$. However, in systems with strongly reduced recombination, it is not yet known whether the reduction is due to a suppressed $k_{\mathrm{f}}$ or improved $k_{\mathrm{d}}$. Finding the answer to this question is crucial for a better understanding of non-Langevin 
recombination and ultimately for obtaining a structure-property relation in this regard - potentially leading to a means to deliver efficient thicker junctions relevant to industrial scaling.

In this work, we address the above question by introducing a procedure which utilizes the principle of detailed balance and accurate characterisation of CT state parameters. We investigated the donoracceptor material system BQR:PC ${ }_{71} \mathrm{BM}$ (benzodithiophene-quaterthiophene-rhodanine:[6,6]-PhenylC71-butyric acid methyl ester) which exhibits a strongly reduced recombination of $\gamma=1 / 2000$ when solvent annealed. We will show that an improved dissociation rate of CT states into free carriers is responsible for the substantial reduction factor in this material system.

\section{Theoretical framework}

The kinetic diagram of a BHJ system is depicted schematically in Figure 1. If we assume that $G_{\mathrm{CT}}$ is the generation rate of $\mathrm{CT}$ states (either directly or via exciton dissociation), then one can write

$$
\frac{d n_{\mathrm{CT}}}{d t}=G_{\mathrm{CT}}-k_{\mathrm{f}} n_{\mathrm{CT}}-k_{\mathrm{d}} n_{\mathrm{CT}}+\beta_{\mathrm{en}} n_{\mathrm{CS}}^{2}
$$

The encounter rate, $\beta_{\mathrm{en}}$, can be slightly reduced relative to $\beta_{\mathrm{L}}$ due to the geometrical confinement of electrons and holes but other morphology-related mechanism can play roles to reduce it also. ${ }^{23}$ Here $k_{\mathrm{d}} n_{\mathrm{CT}}-\beta_{\mathrm{en}} n_{\mathrm{CS}}^{2}$ is the net generation-recombination rate of free charge carriers via CT states. The probability of CT state dissociation into free carriers is commonly described using the Onsager-Braun formalism ${ }^{24,25}$ where

$$
P=\frac{k_{\mathrm{d}}}{k_{\mathrm{d}}+k_{\mathrm{f}}}
$$

is the charge generation quantum yield for the CT states. At steady-state $\frac{d n_{\mathrm{CT}}}{d t}=0$, it then follows from Eq. 2 that $k_{\mathrm{d}} n_{C T}=G_{\mathrm{CT}} P+P \beta_{\mathrm{en}} n_{\mathrm{CS}}^{2}$ from which the net generation-recombination rate of free separated charge carriers is obtained as $k_{\mathrm{d}} n_{\mathrm{CT}}-\beta_{\mathrm{en}} n_{\mathrm{CS}}^{2}=G_{\mathrm{CT}} P-(1-P) \beta_{\mathrm{en}} n_{\mathrm{CS}}^{2}$. At thermal equilibrium $\left(n_{\mathrm{CT}}=n_{\mathrm{CT} \text {,eq }}\right.$ and $n_{\mathrm{CS}}=n_{\mathrm{CS} \text {,eq }}$ ), the net generation-recombination rate of free 
separated charge carriers must be zero, i.e. $G_{\mathrm{CT}, \mathrm{eq}} P=(1-P) \beta_{\mathrm{en}} n_{\mathrm{CS} \text {,eq }}^{2}$. Then, upon eliminating $G_{\mathrm{CT}, \text { eq }}$ in Eq. 2 (note that: $d n_{\mathrm{CT}, \mathrm{eq}} / d t=0$ ), it follows directly that:

$$
\beta_{\mathrm{en}} n_{\mathrm{CS}, \mathrm{eq}}^{2}=k_{\mathrm{d}} n_{\mathrm{CT}, \mathrm{eq}}
$$

while $G_{\mathrm{CT}, \mathrm{eq}}=k_{\mathrm{f}} n_{\mathrm{CT}, e q}$ at thermal equilibrium (cf. Figure 1). ${ }^{26}$

From the perspective of the free charges (see Figure 1), the carrier generation rate $G_{\mathrm{CS}}$ is generally given by $G_{\mathrm{CS}}=G_{\mathrm{CT}} P$, while the (effective) recombination rate is $\beta_{\mathrm{bulk}} n_{\mathrm{CS}}^{2}=(1-P) \beta_{\mathrm{en}} n_{\mathrm{CS}}^{2}$; hence,

$$
\gamma=\frac{\beta_{\mathrm{Bulk}}}{\beta_{\mathrm{L}}}=\gamma_{\mathrm{Geo}} \frac{k_{\mathrm{f}}}{k_{\mathrm{d}}+k_{\mathrm{f}}}=\gamma_{\mathrm{Geo}} \frac{P k_{\mathrm{f}}}{k_{\mathrm{d}}} .
$$

In material systems with high charge generation efficiency $k_{\mathrm{f}} \ll k_{\mathrm{d}}(P \approx 1)$, we therefore expect $\gamma \approx$ $k_{\mathrm{f}} / k_{\mathrm{d}}$, noting that the geometrical confinement of electrons and holes does not play a significant role in the reduction of bimolecular recombination in systems with domain sizes smaller than $10 \mathrm{~nm}$ (such as $\mathrm{BQR}: \mathrm{PC}_{71} \mathrm{BM}^{27}$ ) as shown by Heiber et al. ${ }^{22}$, i.e., $\gamma_{G e o} \sim 1$ (independent of the system). It is thus clear that either increasing $k_{d}$ or decreasing $k_{f}$ can result in an improvement in the charge generation efficiency accompanied by a simultaneous reduction of the bimolecular recombination.

Based on the reciprocity between light absorption and emission, the radiative limit $\left(V_{\mathrm{OC}}^{\mathrm{Rad}}\right)$ and the non-radiative losses $\left(\Delta V_{\mathrm{OC}}^{\mathrm{NR}}\right)$ of the $V_{\mathrm{OC}}$ can be calculated as follows:

$$
\begin{gathered}
q V_{\mathrm{OC}}^{\mathrm{Rad}}=k T \ln \left(\frac{J_{\mathrm{Ph}}^{\mathrm{Rad}}}{J_{0}^{\mathrm{Rad}}}\right) ; \\
\Delta V_{\mathrm{OC}}^{\mathrm{NR}}=V_{\mathrm{OC}}^{\mathrm{Rad}}-V_{\mathrm{OC}}=-\frac{k T}{q} \ln \left(\mathrm{EQE}_{\mathrm{LED}}\right),
\end{gathered}
$$

where $k$ is the Boltzmann constant, $T$ is the absolute temperature, and $\mathrm{EQE}_{\mathrm{LED}}$ is the efficiency of the electroluminescence of the solar cell. Here, $J_{\mathrm{Ph}}$ is the photocurrent under 1 sun illumination given by $J_{\mathrm{Ph}}=q \int_{E_{\min }}^{\infty} \mathrm{EQE}_{\mathrm{PV}} \Phi_{\text {sun }} d E$, and $J_{0}^{\mathrm{Rad}}$ is the radiative dark saturation current defined as $J_{0}^{\mathrm{Rad}}=$ 
$q \int_{E_{\min }}^{\infty} \mathrm{EQE}_{\mathrm{PV}} \Phi_{\mathrm{BB}} d E ; E_{\min }$ is the lower limit of the $b \mathrm{EQE}_{\mathrm{PV}}$ measurement, while $\Phi_{\text {sun }}$ and $\Phi_{\mathrm{BB}}$ are the spectral flux density of the sun and the black body spectra (environment) at room temperature, respectively. Due to the principle of reciprocity, $\Delta V_{\mathrm{OC}}^{\mathrm{NR}}$ can be calculated from $\mathrm{EQE}_{\mathrm{PV}}$ and $\mathrm{EQE} \mathrm{E}_{\mathrm{LED}}$.

On the other hand, given the active layer thickness $d$, the total dark saturation current associated with CT states is equal to the recombination current of free charge carriers at thermal equilibrium and given by:

$$
J_{0}=q \beta_{\mathrm{Bulk}} n_{\mathrm{CS}, \mathrm{eq}}^{2} d,
$$

which is related to the radiative limit of the dark current via $J_{0}=J_{0}^{\mathrm{Rad}} / \mathrm{EQE}_{\mathrm{LED}}$. However, in accordance with Eqs. 3-5 it can be shown that $\beta_{\mathrm{Bulk}} n_{\mathrm{CS}, \mathrm{eq}}^{2}=P k_{\mathrm{f}} n_{\mathrm{CT}, \mathrm{eq}}$. As a result, requiring that $n_{\mathrm{CT}, \mathrm{eq}}=N_{\mathrm{CT}} \exp \left(-E_{\mathrm{CT}} / k T\right)$, Eq. 8 can be rewritten as:

$$
J_{0}^{\mathrm{Rad}}=\mathrm{EQE}_{\mathrm{LED}} \times q d P k_{\mathrm{f}} N_{\mathrm{CT}} \exp \left(-\frac{E_{\mathrm{CT}}}{k T}\right)
$$

where $E_{\mathrm{CT}}$ and $N_{\mathrm{CT}}$ are the energy and density of (excitable) CT states, respectively. From Eq. 9, the reduced decay rate constant $P k_{\mathrm{f}}$ is then obtained as:

$$
P k_{\mathrm{f}}=\frac{J_{0}^{\mathrm{Rad}}}{q d N_{\mathrm{CT}} \mathrm{EQE}_{\mathrm{LED}}} \exp \left(\frac{E_{\mathrm{CT}}}{k T}\right)=\frac{J_{0}^{\mathrm{Rad}}}{q d N_{\mathrm{CT}}} \exp \left(\frac{E_{\mathrm{CT}}+q \Delta V_{\mathrm{OC}}^{\mathrm{NR}}}{k T}\right),
$$

where Eq. 7 was used in the last step. Note that $J_{0}^{\mathrm{Rad}}, E_{\mathrm{CT}}$ and $\Delta V_{\mathrm{OC}}^{\mathrm{NR}}$ can be obtained from experiments. Eq. 10 is a very useful formula because it bridges observable parameters such as $J_{0}^{\mathrm{Rad}}$, $\Delta V_{\mathrm{OC}}^{\mathrm{NR}}$ and $E_{\mathrm{CT}}$ to $k_{\mathrm{f}}$ which are difficult to probe, even using ultra-fast pump-probe techniques in efficient systems. ${ }^{28}$

\section{Device Characterisation}


In Figure 2.a the chemical structure of $B Q R$ and $\mathrm{PC}_{71} \mathrm{BM}$ are shown. Two variations of devices were fabricated from the same active layer solution: (1) the as-cast (AC) device for which no post processing was performed on the spin coated active layer; and (2) the solvent vapor annealed (SVA) device for which the spin coated active layer was exposed to THF vapor followed by thermal annealing. Both AC and SVA devices were fabricated with the previously reported structure ${ }^{29}$ to achieve the optimal device performance. The details of the device fabrication are provided in the Supplementary Information. The current-voltage characteristics $(J-V)$ of 320-nm-thick AC and SVA devices are shown in Figure 2.b. It can be seen that the SVA device has a higher open circuit voltage $\left(V_{\mathrm{OC}}\right)$, higher shortcircuit current $\left(U_{\text {sc }}\right)$ and higher FF and, thus, higher PCE compare to the AC device. As shown by Schwartz et al., even in a 600-nm-thick junction, the PCE remains higher than $8 \%$ when solvent annealed. ${ }^{27}$

Investigation of the morphology of the active layers in AC and SVA devices shows that the BHJ structure is sensitive to the active layer deposition process. Grazing-incidence wide-angle scattering (GIWAX) has revealed that solvent vapour annealing increases the crystallinity and the domain purity in the BQR. This results in the formation of small but pure aggregated domains in the blend for both the donor and the acceptor. ${ }^{27,30}$

Transport and recombination measurements were previously conducted on both AC and SVA devices, as presented in the reference ${ }^{27}$ and also in Figure $\mathbf{S 1}$. The corresponding electron and hole mobilities, measured using the resistance dependent photovoltage (RPV) technqiue, ${ }^{31}$ are summarized in Table 1. Both electron and hole mobilities are improved in SVA devices compare to AC which agrees with the purer domains for both donor and acceptor in the SVA blend. The bimolecular rate coefficient for both devices were measured using transient double injection (DI). ${ }^{32}$ The $\beta_{\text {bulk }}$ for AC devices is 10 times reduced $(\gamma=1 / 10)$, whereas the SVA devices exhibit 2000 times reduced recombination $(\gamma=$ $1 / 2000)$, compared to the Langevin recombination rate coefficient. 


\section{Results and discussion}

In Figure 3.a the sensitive $\mathrm{EQE}_{\mathrm{PV}}$ spectra are shown, where $E_{\min }=1.16 \mathrm{eV}$. The sensitive $\mathrm{EQE}_{\mathrm{PV}}$ were measured using the method presented by Zeiske et $a .^{33}$ from which the sub-gap features, in this case the CT sate parameters, can be measured accurately. The CT state absorption for AC and SVA devices are clearly observed at $\sim 1.4 \mathrm{eV}$. Based on the estimated $J_{0}^{\mathrm{Rad}}$ and $J_{\mathrm{Ph}}, V_{\mathrm{OC}}^{\mathrm{Rad}}$ was calculated using Eq. 6 for both AC and SVA devices. These parameters are plotted versus the lower limit of the integration windows, $E_{\min }$ (Figure 3.b). Furthermore, from the actual cell $V_{\mathrm{OC}}$ we determined $\Delta V_{\mathrm{OC}}^{\mathrm{NR}}$ for both devices (Figure 3.c). Based on Eq. 10, the ratio between the reduced $k_{\mathrm{f}} S$ in SVA and in AC devices can be determined via

$$
\frac{P_{\mathrm{SVA}} k_{\mathrm{f}, \mathrm{SVA}}}{P_{\mathrm{AC}} k_{\mathrm{f}, \mathrm{AC}}}=\frac{J_{0, \mathrm{SVA}}^{\mathrm{Rad}}}{J_{0, \mathrm{AC}}^{\mathrm{Rad}}} \frac{N_{\mathrm{CT}, \mathrm{AC}}}{N_{\mathrm{CT}, \mathrm{SVA}}} \exp \left(\frac{E_{\mathrm{CT}, \mathrm{SVA}}-E_{\mathrm{CT}, \mathrm{AC}}+q\left[\Delta V_{\mathrm{OC}, \mathrm{SVA}}^{\mathrm{NR}}-\Delta V_{\mathrm{OC}, \mathrm{AC}}^{\mathrm{NR}}\right]}{k T}\right) .
$$

While it is very difficult to obtain the number density of CT states $\left(N_{C T}\right)$, the ratio between $N_{\mathrm{CT}} \mathrm{s}$ in AC and SVA device can be estimated from the experimental results.

In order to determine the ratio between $N_{\mathrm{CT}}$ we first extracted the absorption coefficients from the $\mathrm{EQE}_{\mathrm{PV}}$. It has been shown that Beer-Lambert law cannot be applied for this purpose due to cavity induced interference effects. To eliminate the effect of interference we therefore used the procedure proposed by Kaiser et al., ${ }^{34}$ allowing for the sub-gap absorption coefficient spectra to be obtained. This method is based on an iterative inverse transfer matrix formalism for which complex optical constants are required in the visible spectral region. The corresponding refractive indices for $A C$ and SVA devices are provided in the Supplementary Information. The CT state absorption coefficient can be described in terms of (non-adiabatic) Marcus theory ${ }^{35}$ :

$$
\alpha_{\mathrm{CT}}=\frac{f_{\alpha}}{E \sqrt{4 \pi \lambda_{\mathrm{CT}} k T}} \exp \left(-\frac{\left(E_{\mathrm{CT}}+\lambda_{\mathrm{CT}}-E\right)^{2}}{4 \lambda_{\mathrm{CT}} k T}\right)
$$

which parametrises the (weakly coupled) CT state in terms of $E_{\mathrm{CT}}$ which is the energy difference between the ground and excited state of the $\mathrm{CT}$ state, $\lambda_{\mathrm{CT}}$ which is the reorganization energy 
associated with the formation of the $\mathrm{CT}$ state, and $f_{\alpha}$ which is a measure of the strength of the donoracceptor coupling and also proportional to the density of the CT states through $f_{\alpha}=N_{\mathrm{CT}} f_{\sigma}$ in which $f_{\sigma}$ is the $\mathrm{CT}$ states oscillator strength.

In Figure 4 the reduced absorption coefficients $\left(\alpha_{\mathrm{CT}} E\right)$ are shown together with the Gaussian fits based on Eq. 12 and the CT state parameters that have been obtained from the fittings. The pre-factor $f_{\alpha}$ is obtained from the fittings for each system. Here we assume that $f_{\sigma}$ is the same for both $\mathrm{AC}$ and SVA devices, made of the same donor and acceptor molecules, thus $\frac{f_{\alpha, \mathrm{AC}}}{f_{\alpha, \mathrm{SVA}}}=\frac{N_{\mathrm{CT}, \mathrm{AC}}}{N_{\mathrm{CT}, \mathrm{SVA}}}=\frac{250}{33} \pm 1.5$ (see supporting information for the error analysis). In this important step we obtained the ratio between the number density of the interfacial charge transfer states, previously extremely challenging to quantify. Our approach is thus far the only methodology to determine the number density of charge transfer states, however, it is only relative and cannot be applied to compare blends of different donor-acceptor molecules with often unknown oscillator strengths.

From the above analysis, the number density of CT states in the AC system is approximately six times larger than that of the SVA. This agrees with the morphological studies on these systems indicating higher domain purity and larger aggregation in the SVA system, and hence smaller number density of CT states in the SVA films.

Table 1 summarises all the free charge, CT state and thermodynamic parameters required for calculation of $P_{\mathrm{SVA}} k_{\mathrm{f}, \mathrm{SVA}} / P_{\mathrm{AC}} k_{\mathrm{f}, \mathrm{AC}}$ from Eq. 11. From this we find $\frac{P_{\mathrm{SVA}} k_{\mathrm{f}, \mathrm{SVA}}}{P_{\mathrm{AC}} k_{\mathrm{f}, \mathrm{AC}}}=0.23 \pm 0.07$, meaning that the $P k_{\mathrm{f}}$ rate of CT states in the SVA system is approximately 4 times slower than that of AC system. This ratio is not small enough to explain the substantial difference of bimolecular recombination in the two systems $\left(\frac{\gamma_{\mathrm{SVA}}}{\gamma_{\mathrm{AC}}}=0.005 \pm 0.0002\right)$. Using the rationale $\frac{\gamma_{\mathrm{SVA}}}{\gamma_{\mathrm{AC}}}=\frac{P_{\mathrm{SVA}} k_{\mathrm{f}, \mathrm{SVA}}}{P_{\mathrm{AC}} k_{\mathrm{f}, \mathrm{AC}}} \times \frac{k_{\mathrm{d}, \mathrm{AC}}}{k_{d, \mathrm{SVA}}}$ as per Eq. 5, the ratio of CT states dissociation rates can be found to be $\frac{k_{\mathrm{d}, \mathrm{SVA}}}{k_{\mathrm{d}, \mathrm{AC}}} \approx 50 \pm 15$. This implies that the CT states dissociate at a much faster rate in the SVA system than in the AC resulting in significantly reduced recombination. This is the mechanism mainly responsible for the reduced recombination (at 
least in this material system), while reduction in CT state decay rate has a marginal impact on the reduction factor (roughly a factor of 4).

It has been suggested that the dissociation of the CT states at the donor-acceptor interface can be driven by delocalization of charges over a molecule or number of molecules which results in reduced Columbic attraction between electron and hole. ${ }^{36} \mathrm{As}$ a result, the improvement of $\mathrm{CT}$ state dissociation in SVA BQR:PC ${ }_{71} \mathrm{BM}$ can be attributed to the slightly larger and purer domains of the donor and acceptor which can result in more delocalized charges at the interface.

\section{Conclusion}

In summary, we have developed a theoretical/experimental methodology to obtain the number density of $\mathrm{CT}$ states in one system relative to another. The method is generic, with the limitation that the two systems are required to have similar or known CT states oscillator strengths. We have applied this method on the as cast and solvent annealed $B Q R: P C_{71} B M$ solar cells with the latter showing significantly reduced recombination rate constants. Using detailed balance analysis together with accurate characterisation of $\mathrm{CT}$ state and free charge parameters, we have been able to relate the CT state kinetics to the observed strongly suppressed bimolecular recombination of free charges. Our results show that the reduced recombination in SVA devices is due to an improved dissociation rate of CT states upon solvent annealing while a marginal reduction in the CT states decay rate was observed. These results shed considerable light on the nature of reduced bimolecular recombination in $\mathrm{BHJ}$ organic solar cells and is a step towards understanding how to engineer the thicker junctions needed for industrially viable OSCs.

\section{Acknowledgement}

This work was supported by the Sêr Cymru Program through the European Regional Development Fund, Welsh European Funding Office, and Swansea University strategic initiative in Sustainable Advanced Materials. A.A. is a Sêr Cymru II Rising Star Fellow and P.M. a Sêr Cymru II National Research 
Chair. NZ was supported by a studentship through the Sêr Cymru II Program and C.K. is the recipient of a UKRI EPSRC Doctoral Training Account studentship.

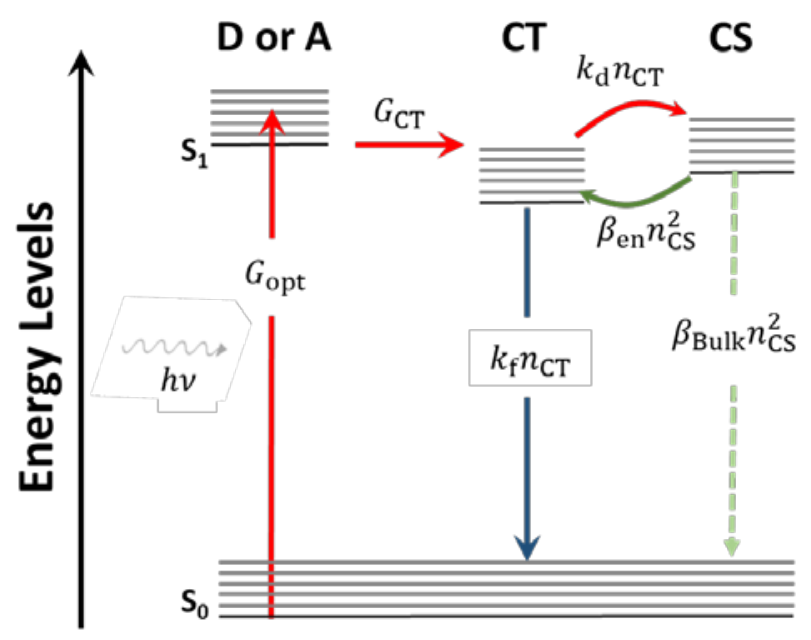

Figure 1. Schematic of charge generation and recombination in a BHJ OSC. The transition illustrated by the dashed downward arrow is the effective recombination rate of separated charge carriers. Note that $k_{\mathrm{d}} n_{\mathrm{CT}}=\beta_{e n} n_{\mathrm{CS}}^{2}$ and $G_{\mathrm{CT}}=k_{\mathrm{f}} n_{\mathrm{CT}}$ at thermal equilibrium. 


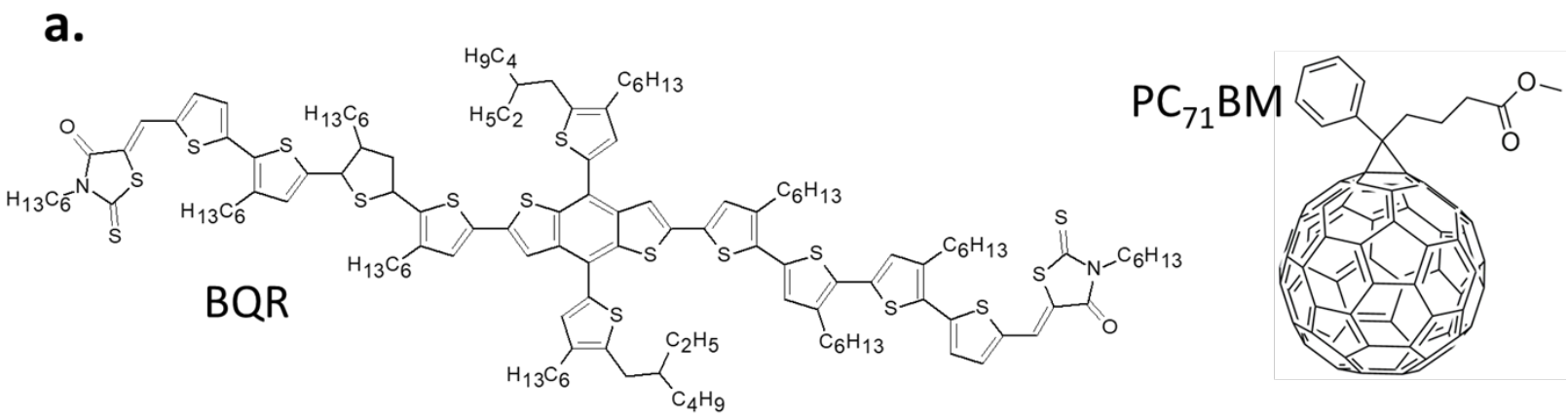

b.

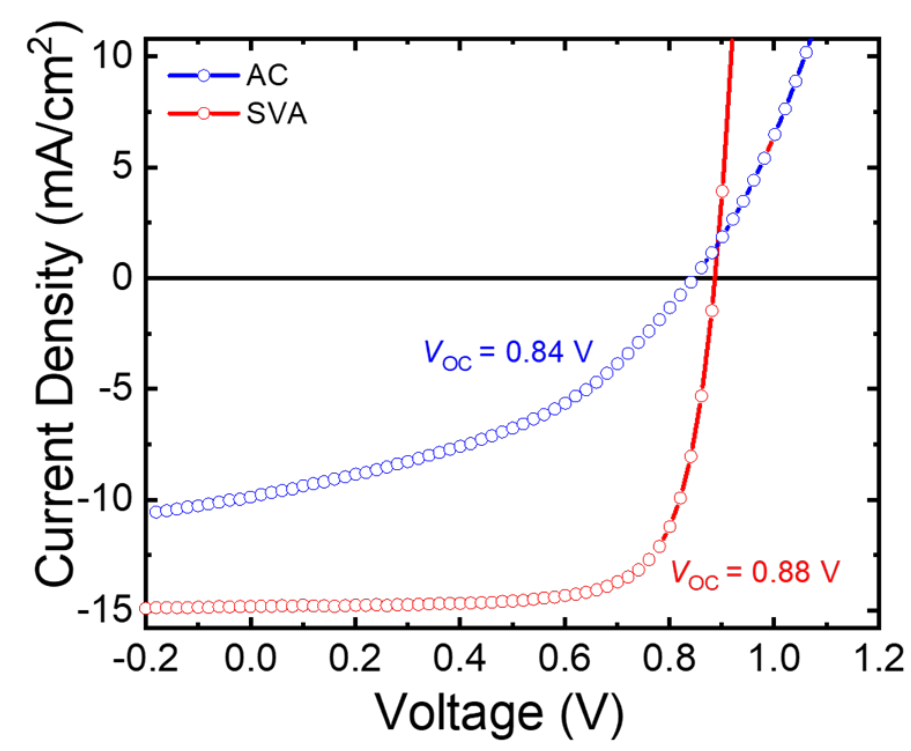

Figure2. (a) Molecular structure of $B Q R$ and $P C_{71} B M$. (b) The $J V$ curves of $A C$ and SVA devices 


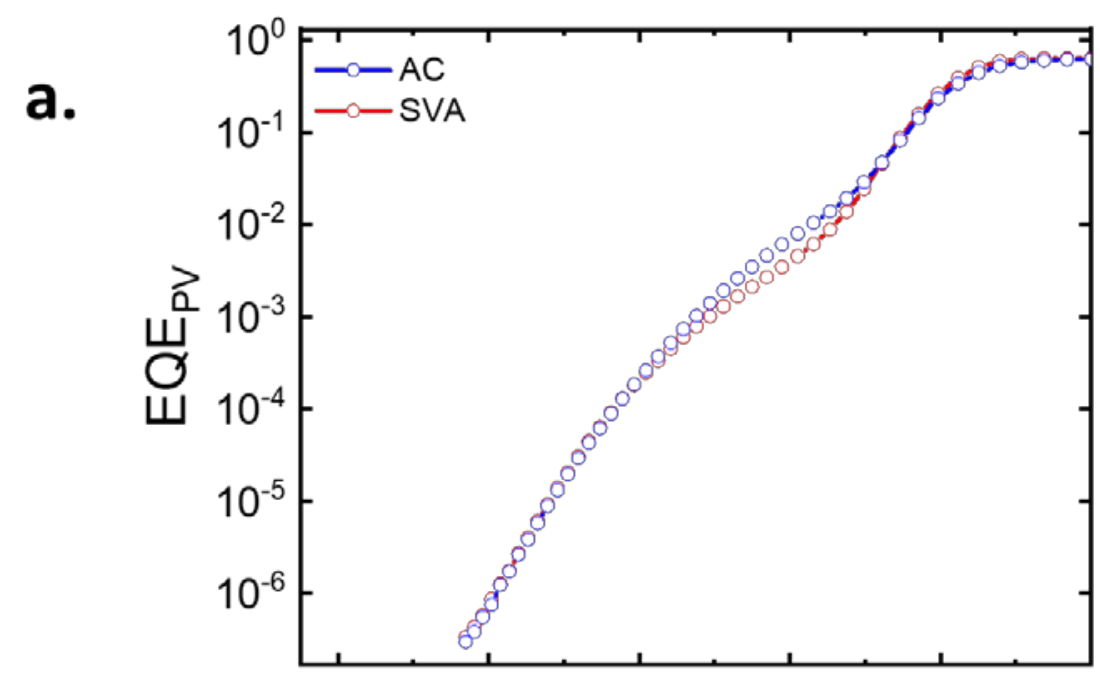

b.

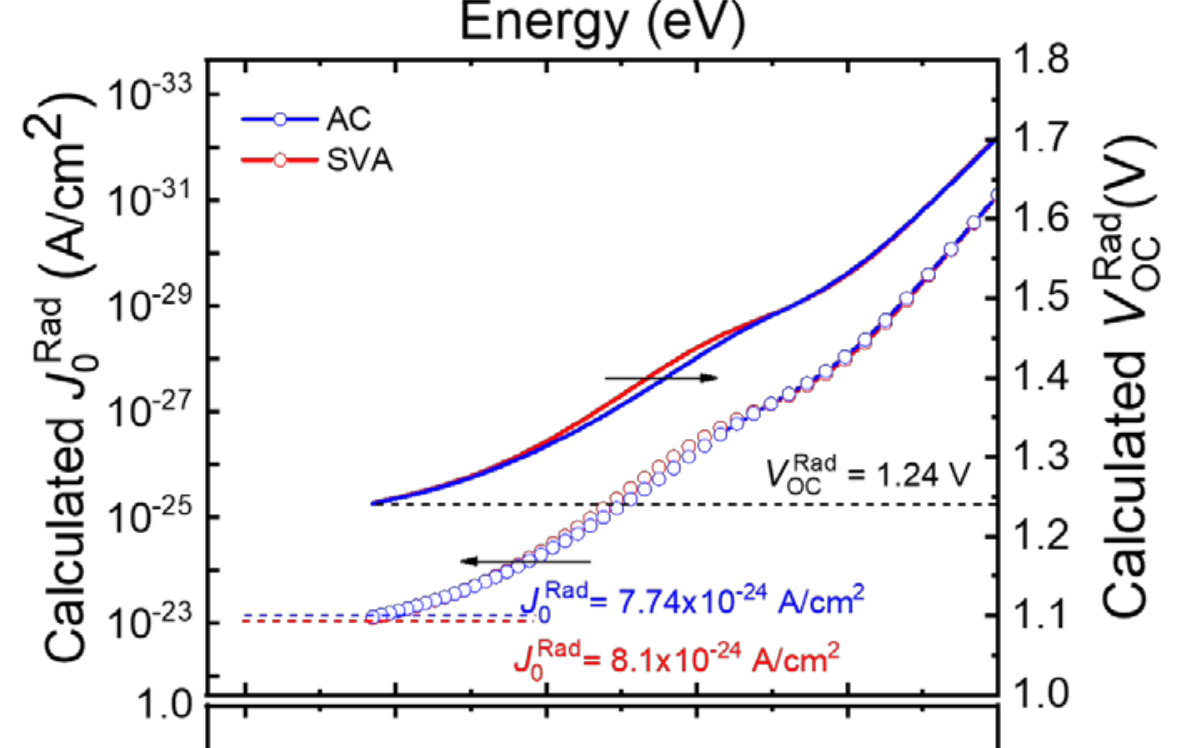

c.

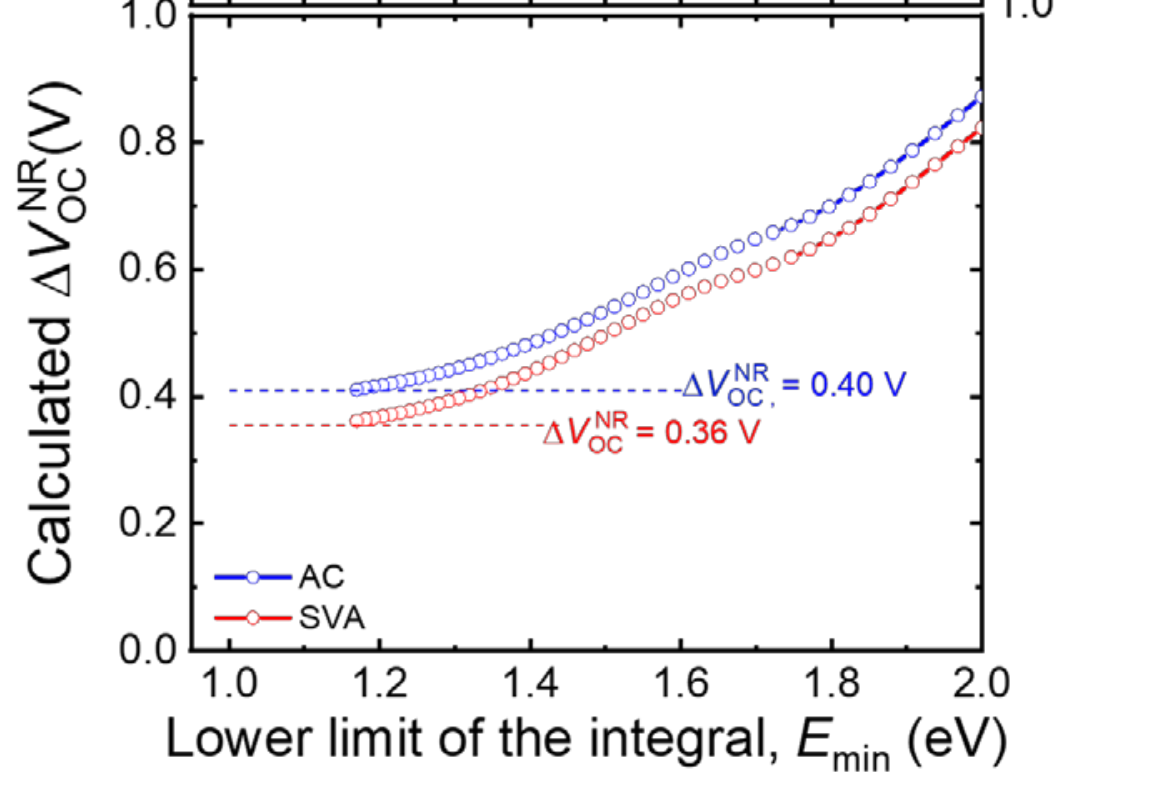


Figure 3. (a) Sensitive $\mathrm{EQE}_{\mathrm{PV}}$ is plotted versus photon energy to show the CT state absorption contribution. (b) $J_{0}^{\mathrm{Rad}}$ and $V_{\mathrm{OC}}^{\mathrm{Rad}}$ and (c) $\Delta V_{\mathrm{OC}}^{\mathrm{NR}}$ for both AC and SVA devices are plotted versus the minimum photon energy at which $J_{0}^{\mathrm{Rad}}$ and hence $V_{\mathrm{OC}}^{\mathrm{Rad}}$ and $\Delta V_{\mathrm{OC}}^{\mathrm{NR}}$ are calculated.

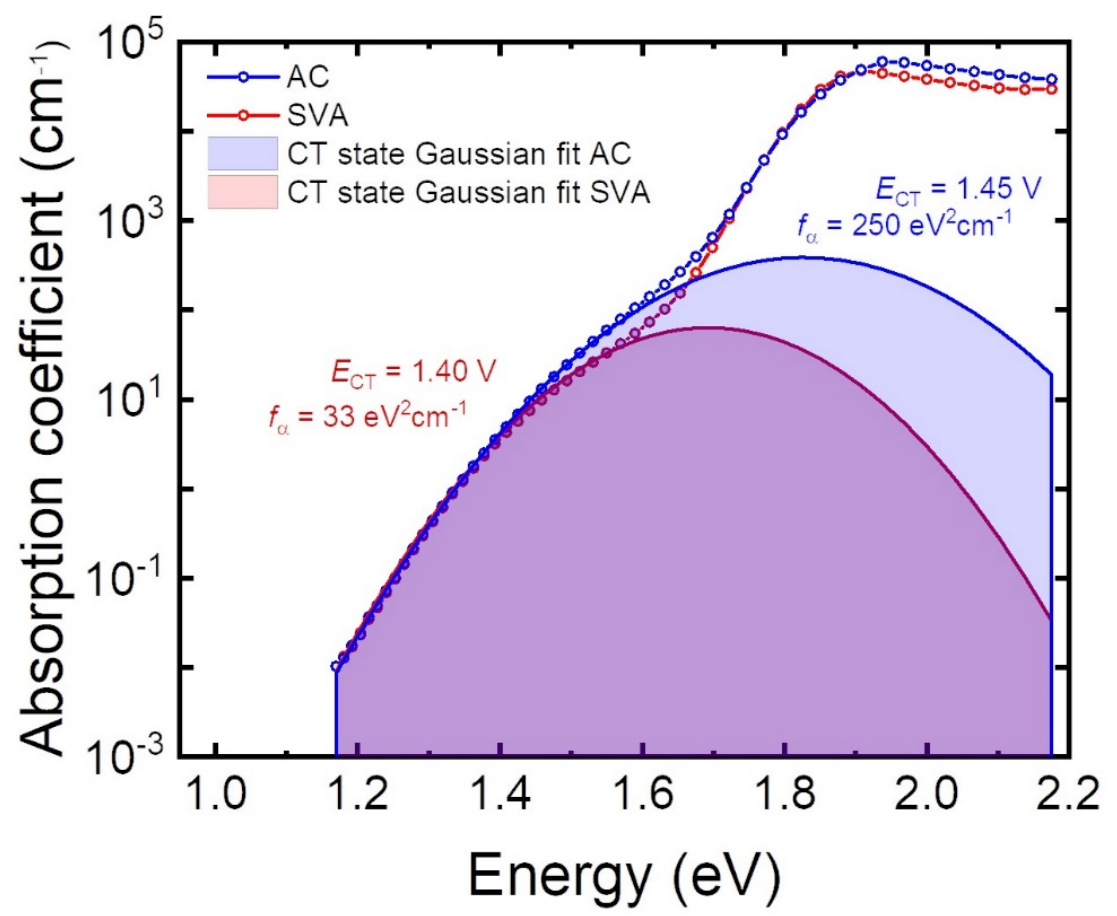

Figure 4. Simulated absorption coefficient for AC and SVA devices plotted versus photon energy. Gaussian fits on the CT state absorption and the CT state parameters obtained from the Gaussian fits are shown.

Table 1. Kinetic and thermodynamic parameters of the as-cast and solvent-annealed BQR:PC71BM.

\begin{tabular}{|c|c|c|c|c|c|c|c|c|c|}
\hline System & $\begin{array}{c}\mu_{e} \\
\left(\mathrm{~cm}^{2} \mathrm{~V}^{-1} \mathrm{~s}^{-1}\right)\end{array}$ & $\begin{array}{c}\mu_{h} \\
\left(\mathrm{~cm}^{2} \mathrm{~V}^{-1} \mathrm{~s}^{-1}\right)\end{array}$ & $\gamma=\frac{\beta_{\text {Bulk }}}{\beta_{\mathrm{L}}}$ & $V_{\mathrm{OC}}(\mathrm{V})$ & $\begin{array}{c}J_{0}^{\mathrm{R}} \\
(\mathrm{A} / \mathrm{cm} 2)\end{array}$ & $\begin{array}{c}\mathrm{Rad} \\
V_{\mathrm{OC}}(\mathrm{V})\end{array}$ & $\begin{array}{c}\mathrm{NR} \\
\Delta V_{\mathrm{OC}}(\mathrm{V})\end{array}$ & $\begin{array}{c}E_{\mathrm{CT}}(\mathrm{eV}) \\
\Delta V_{\mathrm{OC}}^{\mathrm{R}}(\mathrm{V})\end{array}$ \\
\hline AC & $\begin{array}{c}1.5 \times 10^{-3} \\
\pm 2 \times 10^{-5}\end{array}$ & $\begin{array}{c}4 \times 10^{-5} \\
\pm 4.5 \times 10^{-6}\end{array}$ & $\begin{array}{c}0.1 \\
\pm 0.01\end{array}$ & $\begin{array}{c}0.84 \\
\pm 0.004\end{array}$ & $\begin{array}{c}7.74 \times 10^{-24} \\
\pm 10^{-28}\end{array}$ & $\begin{array}{c}1.24 \\
\pm 10^{-7}\end{array}$ & $\begin{array}{c}0.40 \\
\pm 0.004\end{array}$ & $\begin{array}{c}1.45 \\
\pm 0.003\end{array}$ & $\begin{array}{c}0.21 \\
\pm 0.003\end{array}$ \\
\hline SVA & $3 \times 10^{-3}$ & $8 \times 10^{-4}$ & $1 / 2000$ & 0.88 & $8.10 \times 10^{-24}$ & 1.24 & 0.36 & 1.40 & 0.16 \\
$\pm 10^{-28}$ & $\pm 10^{-7}$ & \pm 0.004 & \pm 0.001 \\
\pm 0.001 \\
\hline
\end{tabular}


1. Liu, Q.; Jiang, Y.; Jin, K.; Qin, J.; Xu, J.; Li, W.; Xiong, J.; Liu, J.; Xiao, Z.; Sun, K.; Yang, S.; Zhang, X.; Ding, L., 18\% Efficiency organic solar cells. Science Bulletin 2020, 65 (4), 272-275.

2. Armin, A.; Hambsch, M.; Wolfer, P.; Jin, H.; Li, J.; Shi, Z.; Burn, P. L.; Meredith, P., Efficient, large area, and thick junction polymer solar cells with balanced mobilities and low defect densities. Advanced Energy Materials 2015, 5 (3), 1401221.

3. Meredith, P.; Armin, A., Scaling of next generation solution processed organic and perovskite solar cells. Nat Commun 2018, 9 (1), 5261.

4. Bartesaghi, D.; del Carmen Pérez, I.; Kniepert, J.; Roland, S.; Turbiez, M.; Neher, D.; Koster, L. J. A., Competition between recombination and extraction of free charges determines the fill factor of organic solar cells. Nature communications 2015, 6 (1), 1-10.

5. Lenes, M.; Koster, L. J. A.; Mihailetchi, V. D.; Blom, P. W. M., Thickness dependence of the efficiency of polymer:fullerene bulk heterojunction solar cells. Applied Physics Letters 2006, 88 (24), 243502.

6. Min Nam, Y.; Huh, J.; Ho Jo, W., Optimization of thickness and morphology of active layer for high performance of bulk-heterojunction organic solar cells. Solar Energy Materials and Solar Cells 2010, 94 (6), 1118-1124.

7. Langevin, P., Recombinaison et mobilites des ions dans les gaz. Ann. Chim. Phys 1903, 28 (433), 122.

8. Arkhipov, V.; PEROVA, I. A.; Rudenko, A., Sign-alternating kinetics of radiation-induced current in disordered dielectrics. International journal of electronics 1992, 72 (1), 99-102.

9. Arkhipov, V.; Perova, I., Non-Langevin recombination in disordered dielectrics. Journal of Physics D: Applied Physics 1993, 26 (8), 1301.

10. Shoaee, S.; Armin, A.; Stolterfoht, M.; Hosseini, S. M.; Kurpiers, J.; Neher, D., Decoding Charge Recombination through Charge Generation in Organic Solar Cells. Solar RRL 2019, 3 (11), 1900184.

11. Juška, G.; Arlauskas, K.; Stuchlik, J.; Österbacka, R., Non-Langevin bimolecular recombination in low-mobility materials. Journal of Non-Crystalline Solids 2006, 352 (9-20), 11671171.

12. Jin, H.; Armin, A.; Hambsch, M.; Lin, Q.; Burn, P. L.; Meredith, P., Bulk heterojunction thickness uniformity - a limiting factor in large area organic solar cells? physica status solidi (a) 2015, $212(10), 2246-2254$.

13. Sun, K.; Xiao, Z.; Lu, S.; Zajaczkowski, W.; Pisula, W.; Hanssen, E.; White, J. M.; Williamson, R. M.; Subbiah, J.; Ouyang, J.; Holmes, A. B.; Wong, W. W.; Jones, D. J., A molecular nematic liquid crystalline material for high-performance organic photovoltaics. Nat Commun 2015, 6,6013 .

14. Armin, A.; Subbiah, J.; Stolterfoht, M.; Shoaee, S.; Xiao, Z.; Lu, S.; Jones, D. J.; Meredith, P., Reduced Recombination in High Efficiency Molecular Nematic Liquid Crystalline: Fullerene Solar Cells. Advanced Energy Materials 2016, 6 (22), 1600939.

15. Jin, Y.; Chen, Z.; Dong, S.; Zheng, N.; Ying, L.; Jiang, X. F.; Liu, F.; Huang, F.; Cao, Y., A Novel Naphtho[1,2-c:5,6-c']Bis([1,2,5]Thiadiazole)-Based Narrow-Bandgap pi-Conjugated Polymer with Power Conversion Efficiency Over 10. Adv Mater 2016, 28 (44), 9811-9818.

16. Armin, A.; Chen, Z.; Jin, Y.; Zhang, K.; Huang, F.; Shoaee, S., A Shockley-Type Polymer: Fullerene Solar Cell. Advanced Energy Materials 2018, 8 (7), 1701450.

17. Li, G.; Shrotriya, V.; Yao, Y.; Yang, Y., Investigation of annealing effects and film thickness dependence of polymer solar cells based on poly(3-hexylthiophene). Journal of Applied Physics 2005, 98 (4), 043704.

18. Sandberg, O. J.; Zeiske, S.; Zarrabi, N.; Meredith, P.; Armin, A., Charge Carrier Transport and Generation via Trap-Mediated Optical Release in Organic Semiconductor Devices. Physical Review Letters 2020, 124 (12), 128001.

19. Tvingstedt, K.; Deibel, C., Temperature dependence of ideality factors in organic solar cells and the relation to radiative efficiency. Advanced Energy Materials 2016, 6 (9), 1502230. 
20. Koster, L. J. A.; Mihailetchi, V. D.; Blom, P. W. M., Bimolecular recombination in polymer/fullerene bulk heterojunction solar cells. Applied Physics Letters 2006, 88 (5), 052104.

21. Groves, C.; Greenham, N. C., Bimolecular recombination in polymer electronic devices. Physical Review B 2008, 78 (15), 155205.

22. Heiber, M. C.; Baumbach, C.; Dyakonov, V.; Deibel, C., Encounter-limited charge-carrier recombination in phase-separated organic semiconductor blends. Phys Rev Lett 2015, 114 (13), 136602.

23. Gorenflot, J.; Heiber, M. C.; Baumann, A.; Lorrmann, J.; Gunz, M.; Kämpgen, A.; Dyakonov, V.; Deibel, C., Nongeminate recombination in neat P3HT and P3HT:PCBM blend films. Journal of Applied Physics 2014, 115 (14), 144502.

24. Braun, C. L., Electric field assisted dissociation of charge transfer states as a mechanism of photocarrier production. The Journal of chemical physics 1984, 80 (9), 4157-4161.

25. Koster, L. J.; Smits, E.; Mihailetchi, V.; Blom, P., Device model for the operation of polymer/fullerene bulk heterojunction solar cells. Physical Review B 2005, 72 (8), 085205.

26. Sandberg, O. J.; Armin, A., On the effect of surface recombination in thin film solar cells, light emitting diodes and photodetectors. Synthetic Metals 2019, 254, 114-121.

27. Schwarz, K. N.; Geraghty, P. B.; Mitchell, V. D.; Khan, S. U.; Sandberg, O. J.; Zarrabi, N.; Kudisch, B.; Subbiah, J.; Smith, T. A.; Rand, B. P.; Armin, A.; Scholes, G. D.; Jones, D. J.; Ghiggino, K. P., Reduced Recombination and Capacitor-like Charge Buildup in an Organic Heterojunction. J Am Chem Soc 2020, 142 (5), 2562-2571.

28. Armin, A.; Durrant, J. R.; Shoaee, S., Interplay Between Triplet-, Singlet-Charge Transfer States and Free Charge Carriers Defining Bimolecular Recombination Rate Constant of Organic Solar Cells. The Journal of Physical Chemistry C 2017, 121 (25), 13969-13976.

29. Geraghty, P. B.; Lee, C.; Subbiah, J.; Wong, W. W.; Banal, J. L.; Jameel, M. A.; Smith, T. A.; Jones, D. J., High performance p-type molecular electron donors for OPV applications via alkylthiophene catenation chromophore extension. Beilstein J Org Chem 2016, 12, 2298-2314.

30. Bourque, A. J.; Engmann, S.; Fuster, A.; Snyder, C. R.; Richter, L. J.; Geraghty, P. B.; Jones, D. J., Morphology of a thermally stable small molecule OPV blend comprising a liquid crystalline donor and fullerene acceptor. Journal of Materials Chemistry A 2019, 7 (27), 16458-16471.

31. Stolterfoht, M.; Philippa, B.; Armin, A.; Pandey, A. K.; White, R. D.; Burn, P. L.; Meredith, P.; Pivrikas, A., Advantage of suppressed non-Langevin recombination in low mobility organic solar cells. Applied Physics Letters 2014, 105 (1), 013302.

32. Armin, A.; Juska, G.; Philippa, B. W.; Burn, P. L.; Meredith, P.; White, R. D.; Pivrikas, A., Doping-Induced Screening of the Built-in-Field in Organic Solar Cells: Effect on Charge Transport and Recombination. Advanced Energy Materials 2013, 3 (3), 321-327.

33. Zeiske, S.; Kaiser, C.; Meredith, P.; Armin, A., Sensitivity of Sub-Bandgap External Quantum Efficiency Measurements of Solar Cells under Electrical and Light Bias. ACS Photonics 2019, 7 (1), 256-264.

34. Kaiser, C.; Zeiske, S.; Meredith, P.; Armin, A., Determining Ultralow Absorption Coefficients of Organic Semiconductors from the Sub-Bandgap Photovoltaic External Quantum Efficiency. Advanced Optical Materials 2019, 8 (1), 1901542.

35. Vandewal, K.; Tvingstedt, K.; Gadisa, A.; Inganäs, O.; Manca, J. V., Relating the open-circuit voltage to interface molecular properties of donor:acceptor bulk heterojunction solar cells. Physical Review B 2010, 81 (12), 125204.

36. Bakulin, A. A.; Rao, A.; Pavelyev, V. G.; van Loosdrecht, P. H.; Pshenichnikov, M. S.; Niedzialek, D.; Cornil, J.; Beljonne, D.; Friend, R. H., The role of driving energy and delocalized states for charge separation in organic semiconductors. Science 2012, 335 (6074), 1340-1344. 